\title{
Метод и Программа Обработки Распределенных Электронных Платежей в Среде Облачных Вычислений
}

\author{
Алекпер Алиев ${ }^{1}$ Р Рамин Самедов ${ }^{2}$ \\ ${ }_{1,2}$ Бакинский Государственный Университет, Баку, Азербайджан \\ 1aaliyev@mail.ru, ramin.samedov@gmail.com
}

\begin{abstract}
Аннотация- В данной статье дано определение электронных платежей, описано проблема обработки параллельно независимо друг от друга инициирующих транзакций в среде облачных вычислений. Для решения предложен метод и программа обработки распределенных электронных платежей в среде облачных вычислений.
\end{abstract}

Ключевые слова- среда облачных вычислений, электронные платежи, обработка транзакций, распределенные платежи

\section{I. ВВЕДЕНИЕ}

С каждым днем все большую популярность получают среды облачных вычислений. Эта технология является шлюзом для генерации, анализа и обработки данных. Облачные вычисления (Cloud computing) представляют собой модель обработки информации, при которой как аппаратные, так и программные ресурсы, задействованные в процессе решения задачи, предоставляются пользователям как онлайн - сервис. Основным преимуществом использования облака является скрытие сложной инфраструктуры, обеспечивающей доступность информации и средств её обработки (программного обеспечения), от конечного пользователя. Это позволяет им сосредоточиться на выполнении своих функциональных обязанностей, не задумываясь о нюансах технологии обработки информации [1].

Среда облачных вычислений представляет собой распределенную систему обработки информации, где множество территориально удаленных друг от друга узлов, объединены системой передачи данных и взаимодействуют посредством обмена сообщениями.

При обработке электронных платежей в среде облачных вычислений есть большое количество клиентов одновременно, параллельно независимо друг от друга инициирующих платежи. В подобных системах определить инициатора, звено фиксации транзакции и менеджера обработки данных не является легкой задачей. При обработке платежей, в случаи неуспешных транзакций, обязательно необходимо успешно возвращать средства клиентов, которые инициировали транзакции.

Для обработки такого большого количество параллельных, распределенных операций необходима хорошо организованная модель обработки распределённых электронных платежей в среде облачных вычислений.
А. Транзактная обработка информациии в распределенных системах

Системы распределенной обработки, или распределенные системы (РС), функционирующие в компьютерных сетях, является одной из наиболее перспективных и быстро развивающихся областей информатики. Такое место они заняли благодаря их существенным преимуществам по сравнению с изолированными системами, функционирующими на базе отдельных компьютеров. Наиболее успешным и часто применяемым на практике типом РС является распределенная база данных (РБД), представляющая собой интеграцию автономных локальных баз данных, географически распределенных и связанных посредством компьютерной сети. Все локальные БД предполагаются изначально целостными и непротиворечивыми. Узлы взаимодействуют между собой путем обмена сообщениями. Средством взаимодействия пользователя с РБД являются транзакции. Транзакцией называется последовательность операций (подтранзакций) над РБД, переводящая ее из одного непротиворечивого состояния в другое согласованное состояние. Каждая подтранзакция перед началом своей работы должна захватить в каждом узле ресурс. Две транзакции вступают в конфликт тогда и только тогда, когда они работают с одним и тем же общим ресурсом и, по крайней мере, одна из реализуемых ими операций является записью. Порядок выполнения действий двух транзакций существен только в том случае, если они конфликтуют. Выполнение каждой отдельной транзакции сохраняет целостное состояние РБД. Следовательно, несколько последовательно выполняемых транзакций также сохраняют целостное состояние РБД. Однако при параллельной работе этих транзакций содержимое РБД может оказаться нецелостным. Главной проблемой при параллельном выполнении транзакции является обеспечение целостности информации в распределенных системах.

В распределенных системах должен быть реализован надежный широковещательный режим передачи сообщений. Предельное время доставки любого сообщения из одного узла в другой узел ограничено физическими возможностями канала связи. В каждом узле имеется служба времени, являющаяся частью службы единого времени РС. В каждом узле могут быть 
инициированы и параллельно выполнены несколько транзакций. В узлах РC размещено множество информационных ресурсов. К каждому ресурсу формируется очередь транзактных запросов. Для обеспечения надежного внесения изменений в базу данных и поддержания непротиворечивого состояния распределенной базы данных в каждом узле используется журнал транзакций, в котором фиксируется информация, достаточная для внесения изменений в базу данных и для анулирования под транзакции при откате [2].

\section{II. ЭЛЕКТРОННЫЕ ПЛАТЕЖИ В СРЕДЕ ОБЛАЧНЫХ ВЫЧИСЛЕНИЙ}

Электронная платёжная система - это система расчётов между финансовыми организациями, бизнес организациями, мерчантами (электронный магазин) и Интернет пользователями при покупке-продаже товаров через Интернет. Естественно с развитием века интернета и мобильных приложений, значимость электронных платежей растет. Обработка электронных платежей является одной из сложных и важных задач. Такую сложную задачу, как обработка электронных платежей часто передают среде облачных вычислений.

Во время совершения электронных платежей в среде облачных вычислений, могут возникнуть ситуации, при которых операция, совершаемая клиентом, не завершится, но средства у клиента спишутся. Основная причина подобной ситуации заключается в том, что количество инициаторов электронных платежей большое, но при этом один и тот же инициатор транзакций может создать новую операцию, не завершив первую операцию.

Среда облачных вычислений имеет ограниченное количество узлов, обрабатывающих транзакции. Также при обработке электронных платежей появляются два дополнительных узла кроме среды облачных вычислений. Это международные платежные системы VISA или MASTERCARD, а также банк карт держателя, у которого в конечном итоге и происходит списание средств [3].

\section{III. МЕТОД ОБРАБОТКИ РАСПРЕДЕЛЕННЫХ ЭЛЕКТРОННЫХ ПЛАТЕЖЕЙ}

Для обработки распределенных электронных платежей предлагется следующий метод: в среде облачных вычислений создается механизм учета всех распределенных электронных платежей, при помощи которого клиенты совершающие электронные платежи в случае тех или иных ошибок гарантированно получат свои средства обратно. Для этого создается набор правил обмена сообщениями между мерчантом и средой облачных вычислений, а также между средой облачных вычислений и международными платежными системами. Вследствие этого получается, что мерчант является инициатором транзакций, и назовём его TG (transaction generator). Среда облачных вычислений является звеном фиксации и начала транзакции, и назовем её ТМ (transaction manager), a международные платежные системы VISA или MASTERCARD назовем DM (data manager) - менеджер обработки данных.
Ниже на рисунке1 отображена схема метода работы узлов со средой облачных вычислений для обработки электронных платежей.

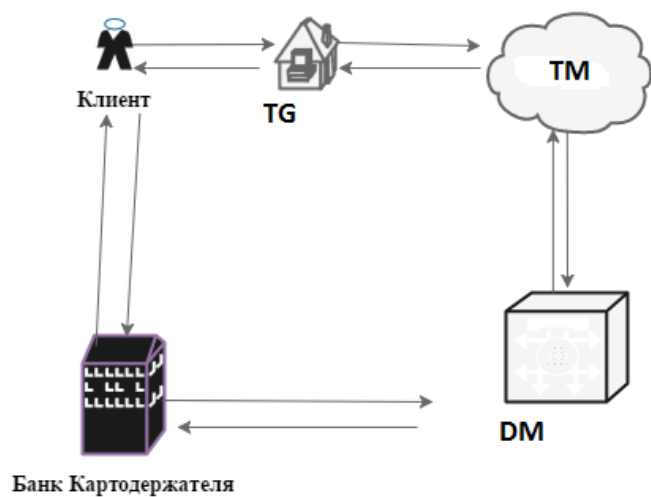

Рисунок 1. Схема работы узлов со средой облачных вычислений

Как видно по рисунку 1 клиент подключившись к сайту мерчанта, выбирает продукты и пытается совершить платеж, отправляет сообщения о покупке на модуль TG. TG генерирует соответствующую транзакцию, присвоив атрибуты транзакций, формирует и передает дескрипторы транзакций в модуль ТМ. Модуль ТМ инициирует и начинает транзакцию, присвоив ей уникальный идентификатор. Модуль ТМ генерирует запрос к модулю DM на списывания средств с клиента, после чего уведомляет модуль ТМ о результате.

\section{IV. ЭКСПЕРИМЕНТ}

Для проведения эксперимента метода обработки распределенных электронных платежей в среде облачных вычислений предлагется выполнить следующий программный код:

CREATE OR REPLACE PACKAGE BODY CLOUD.PAYMENTS_MSG AS

\section{/*----------------}

TYPE $t$ CLOUD MASTER TAB IS TABLE OF CLOUD_MASTER\%ROWTYPE;

$$
\begin{aligned}
& \text {-- Блок констант } \\
& \text { c_Bank_Swift_Code Varchar2(20) := 'AAZYYQ22'; -- Swift Code }
\end{aligned}
$$
Banka

c_Oracle_Dir_Name_UPL Varchar2(20):=

'CLOUDXML_FILE_UPL'; -- Директория Oracle для файла

c_Oracle_Dir_Name_DISP Varchar2(20):=

'CLOUDXML_FILE_DISP'; -- Директория Oracle для файла

$$
\text { c_Oracle_Dir_Name_OUT Varchar2(20):= }
$$

'CLOUDXML_FILE_OUT'; -- Директория Oracle для файла

c_Tag_Batch_Begin Varchar2(10); -- :20: -- TAG в txt сообщение (начало batch сообщения) 


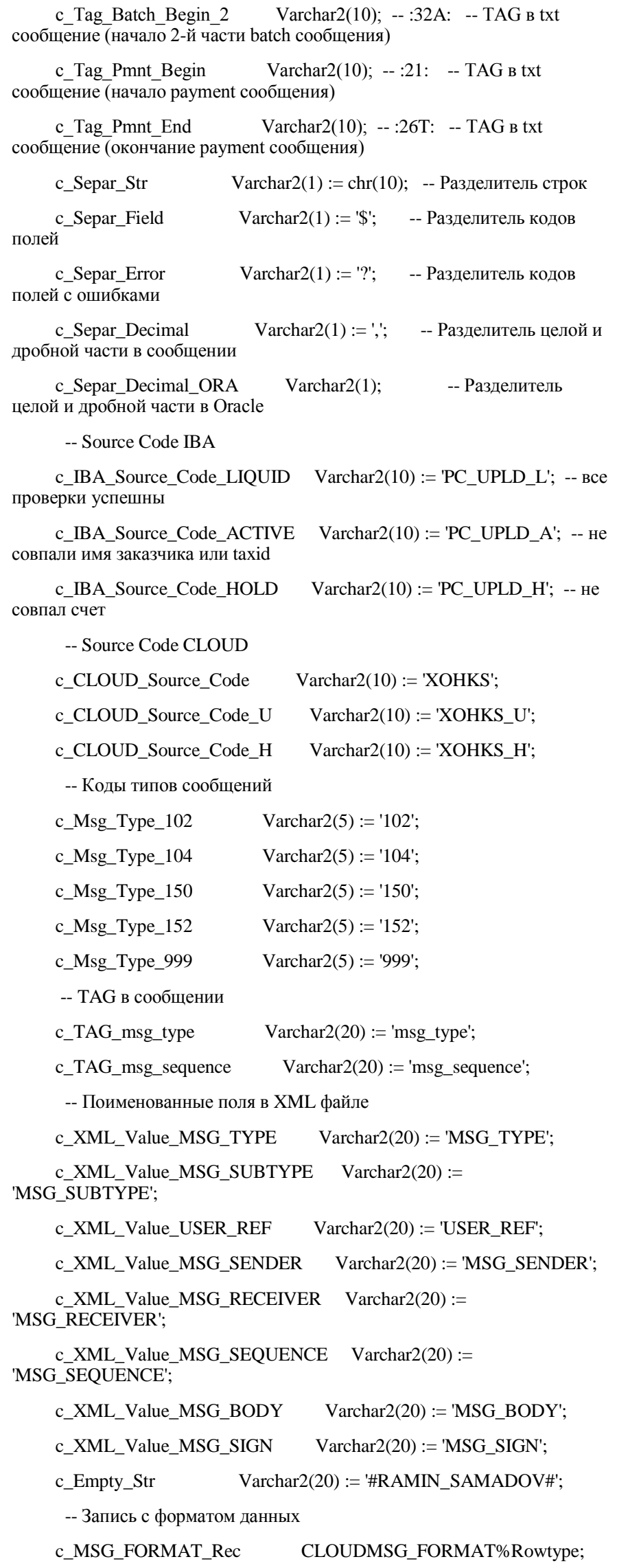

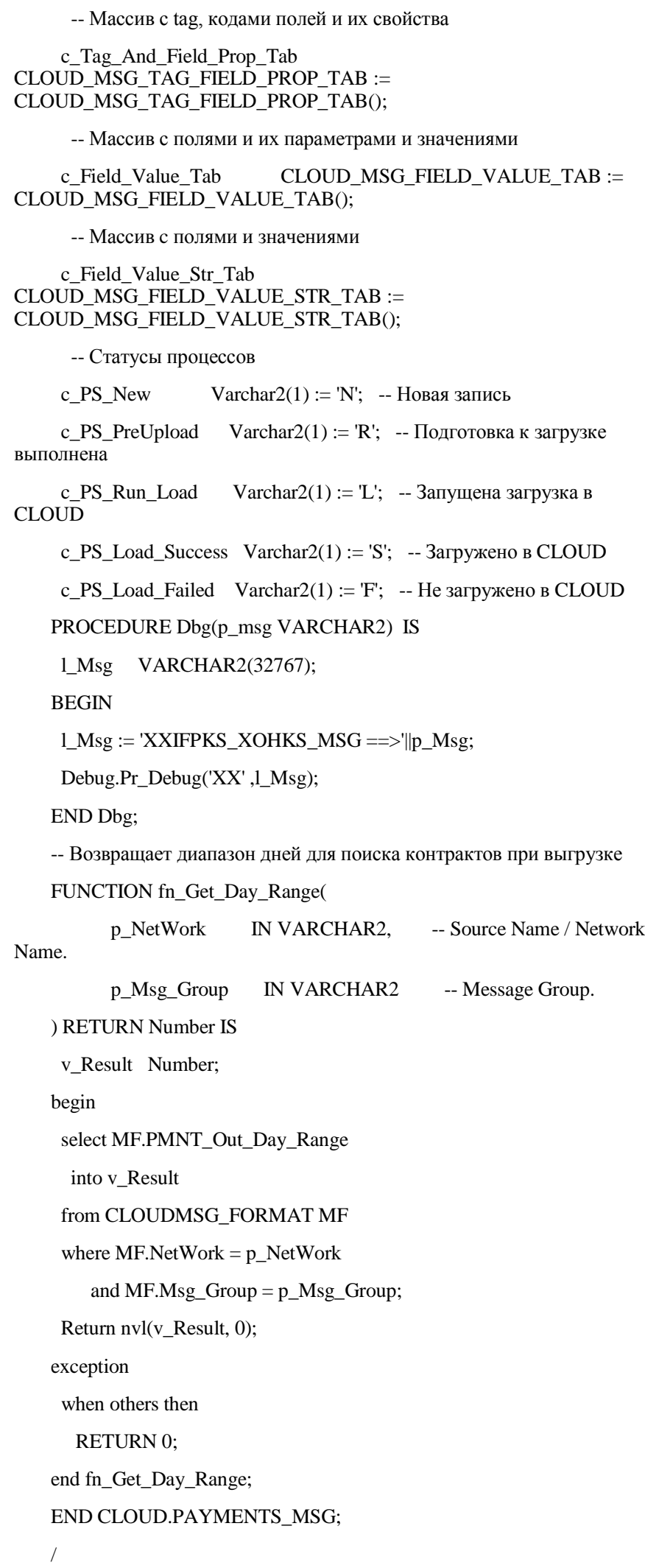

-- Возвращает диапазон дней для поиска контрактов при выгрузке FUNCTION fn_Get_Day_Range(

P_NetWork $\quad$ IN VARCHAR2,
p_Msg_Group $\quad$ IN VARCHAR2
)
RETURN Number IS
v_Result Number;
begin

select MF.PMNT_Out_Day_Range

into v_Result

from CLOUDMSG_FORMAT MF

where MF.NetWork = p_NetWork

and MF.Msg_Group = p_Msg_Group;

Return nvl(v_Result, 0);

exception

when others then

RETURN 0;

end fn_Get_Day_Range;

END CLOUD.PAYMENTS_MSG;

/

\section{ЗАКЛЮЧЕНИЕ}

В результате работы метода обработки распределенных электронных платежей в среде облачных вычислений был получен успешный результат эффективно 
обрабатывающий параллельные транзакции. В данном методе среда облачных вычислений инициировала транзакцию, в успешном случае завершала ее, в противном случае откатывала по запросу или по тайм ауту, держа постоянно систему в согласованном состоянии. Проведенный эксперимент, показал эффективность данного метода. В отличие от других методов, данный метод позволяет параллельно обрабатывать транзакции, в отличии от других методов, где в момент обработки транзакции другие платежи не могут пройти. Таким образом, данный метод позволяет эффективно и более производительно обрабатывать параллельные транзакции.

\section{ЛИТЕРАТУРА}

[1] А.А.Алиев, Р.Б.Самедов, «Разработка алгоритма обработки распределенных электронных платежей в среде облачных вычислений», Системы управления и информационные технологии, научно-технический журнал, перспективные исследования, Воронеж, 2016, vol.66, № 4.1, c.110-113.

[2] P.Buxmann, T.Hess, Di.S.Lehmann, "Software as a Service," Wirtschaftsinformatik, 2008, № 50.6., pp. 500-503.

[3] G.Schneider, "Electronic Commerce," 12th Edition of Course Technology, 2016, 598. p. 